Claremont Colleges

Scholarship@ Claremont

All HMC Faculty Publications and Research

HMC Faculty Scholarship

11-1-1997

\title{
Borsuk-Ulam Implies Brouwer: A Direct Construction
}

Francis E. Su

Harvey Mudd College

\section{Recommended Citation}

Francis Edward Su. "Borsuk-Ulam implies Brouwer: a direct construction." Amer. Math. Monthly, 104(9):855-859, 1997.

This Article is brought to you for free and open access by the HMC Faculty Scholarship at Scholarship @ Claremont. It has been accepted for inclusion in All HMC Faculty Publications and Research by an authorized administrator of Scholarship @ Claremont. For more information, please contact scholarship@cuc.claremont.edu. 
2. D. H. Bailey, P. Borwein, and S. Plouffe, On the rapid computation of various polylogarithmic constants, Mathematics of Computation (to appear).

3. D. H. Bailey and S. Plouffe, Recognizing numerical constants, Proceedings of the Workshop on Organic Mathematics (electronic at www.cecm.sfu.ca/organics/).

4. H. R. P. Ferguson, D. H. Bailey, and S. Arno, Analysis of PSLQ, an integer relation finding algorithm, forthcoming.

5. M. Petkovšek, H. S. Wilf, and D. Zeilberger, $A=B$, A K Peters, Wellesley, Mass., 1996.

Wolfram Research, Inc.

Macalester College

100 Trade Center Drive

St. Paul, MN 55105

Champaign, IL 61820

wagon@macalester.edu

victor@wolfram.com

\section{Borsuk-Ulam Implies Brouwer: A Direct Construction}

\section{Francis Edward Su}

1. INTRODUCTION. The Borsuk-Ulam theorem and the Brouwer fixed point theorem are well-known theorems of topology with a very similar flavor. Both are non-constructive existence results with somewhat surprising conclusions. Most topology textbooks that cover these theorems (e.g., [4], [5], [6]) do not mention the two are related - although, in fact, the Borsuk-Ulam theorem implies the Brouwer Fixed Point Theorem.

The theorems themselves are often proved using the machinery of algebraic topology or the concept of degree of a map. That one theorem implies the other can therefore be established once one understands this machinery, but this requires background. Moreover, such proofs tend to be indirect, relying on the equivalence of these existence theorems with corresponding non-existence theorems. For instance, Dugundji and Granas [3] show that the Borsuk-Ulam theorem is equivalent to the statement that no antipode-preserving, continuous map $f$ : $S^{n} \rightarrow S^{n}$ can be homotopic to a constant map. From this one can see that the Brouwer fixed point theorem is a special case, because it can be shown equivalent to the statement that the identity map $i d: S^{n} \rightarrow S^{n}$ (which is antipode-preserving) is not homotopic to a constant map.

However, such an indirect approach is not really necessary, and perhaps a more direct proof would give insight as to how the two theorems are related. The purpose of this note is to provide a completely elementary proof that the BorsukUlam theorem implies the Brouwer theorem by a direct construction, in which the existence of antipodal points in one theorem yields the asserted fixed point in the other.

2. THE THEOREMS. Let $S^{n}$ denote the unit $n$-sphere in $\mathbb{R}^{n+1}$, i.e., all points at distance one from the origin. Two points are antipodal if they lie opposite each other on the sphere-i.e., $\{\mathbf{x},-\mathbf{x}\}$ for some $\mathbf{x}$. 
The Borsuk-Ulam Theorem. Let $f: S^{n} \rightarrow \mathbb{R}^{n}$ be a continuous map. There exists a pair of antipodal points on $S^{n}$ that are mapped by $f$ to the same point in $\mathbb{R}^{n}$.

This theorem was conjectured by S. Ulam and proved by K. Borsuk [1] in 1933. In particular, it says that if $f=\left(f_{1}, f_{2}, \ldots, f_{n}\right)$ is a set of $n$ continuous real-valued functions on the sphere, then there must be antipodal points on which all the functions agree. For instance, one interpretation for the case $n=2$ is that there is always a pair of antipodal points on the earth's surface with the same temperature and barometric pressure (assuming, of course, that temperature and pressure vary continuously).

Let $B^{n}$ denote the unit $n$-ball in $\mathbb{R}^{n}$. A fixed point for a map $f$ from a space into itself is a point $\mathbf{y}$ such that $f(\mathbf{y})=\mathbf{y}$. The following theorem, due to L.E.J. Brouwer, is one of the most celebrated theorems in topology:

The Brouwer Fixed Point Theorem. Every continuous map $f: B^{n} \rightarrow B^{n}$ possesses a fixed point.

Brouwer proved the case $n=3$ in 1909, and Hadamard followed in 1910 with a proof for all dimensions. Brouwer gave a different proof in 1912 [2]. See [3] for more historical notes and a survey of fixed point theory.

In dimension three, the Brouwer theorem is often interpreted as follows: no matter how you slosh around the coffee in a coffee cup (as long as you do it continuously), some point is always in the same position it was before the sloshing took place (although it might have moved around in the meantime). Moreover, should you try to move this point out of its original position, you will unavoidably move some other point back into its original position.

3. THE IDEA. As motivation we first briefly sketch a construction that shows how the Borsuk-Ulam theorem implies the Brouwer fixed point theorem.

We choose to think of $B^{n}$ as $[-1,1]^{n}$, the " $n$-cube" in $\mathbb{R}^{n}$. Similarly, we choose to think of $S^{n}$ as the boundary of the $(n+1)$-cube $[-1,1]^{n+1}$ in $\mathbb{R}^{n+1}$ :

$$
S^{n}=\left\{\mathbf{x}\left|\mathbf{x}=\left(x_{1}, x_{2}, \ldots, x_{n+1}\right), \quad\right| x_{i} \mid \leq 1 \text { and } \max \left|x_{i}\right|=1\right\} .
$$

The "cubical" $n$-sphere is homeomorphic to the usual $n$-sphere via the rays from the origin. In fact, this is an antipode-preserving homeomorphism, so the BorsukUlam theorem holds for maps on cubical $n$-spheres. We choose to work with cubical $n$-spheres and $n$-balls because constructing and describing functions on such objects is easier in rectangular coordinates.

Given $f: B^{n} \rightarrow B^{n}$, we would like to construct a map $g: S^{n} \rightarrow \mathbb{R}^{n}$ that encodes $f$ in such a manner that the existence of Borsuk-Ulam antipodal points for $g$ implies the existence of a Brouwer fixed point for $f$.

The idea is as follows: on the cubical $n$-sphere, the "top" and "bottom" faces of the cube are homeomorphic copies of $B^{n}$. These are separated by an "equatorial" band, consisting of the other faces. Our task is to define a continuous function $g$ on these three regions of $S^{n}$. On the top face, we define $g$ in such a way that a zero of $g$ implies a fixed point for $f$. We then define $g$ on the bottom face so that the image of each point there is the negative of the image of its antipode on the top face. Such map is called antipode-preserving-meaning $g(-\mathbf{x})=-g(\mathbf{x})$. If we can patch-in the equatorial region with a map that is also antipode-preserving but never zero, then the Borsuk-Ulam Theorem guarantees the existence of antipodal 
points that get mapped by $g$ to the same point. Because $g$ is antipode-preserving, these antipodal points must get mapped to zero, which by construction cannot occur in the equatorial band. A zero for $g$ on the top or bottom face then implies a fixed point for $f$.

4. THE CONSTRUCTION. We seek to construct a map $g=\left(g_{1}, g_{2}, \ldots, g_{n}\right)$ : $S^{n} \rightarrow \mathbb{R}^{n}$ that is continuous and antipode-preserving, i.e., $g(-\mathbf{x})=-g(\mathbf{x})$.

We first construct $g$ on the "top" and "bottom" faces. Note that each face, on which some coordinate $x_{k}= \pm 1$, is an $n$-cube. When $x_{n+1}= \pm 1$ we obtain:

$$
S_{\text {top }}^{n}=\left\{\mathbf{x} \mid \mathbf{x}=\left(x_{1}, x_{2}, \ldots, x_{n}, 1\right)\right\} \quad \text { and } \quad S_{\text {bot }}^{n}=\left\{\mathbf{x} \mid \mathbf{x}=\left(x_{1}, x_{2}, \ldots, x_{n},-1\right)\right\} \text {, }
$$

which denote the top and bottom faces of the cubical $S^{n}$. See Figure 1. Let $p: \mathbb{R}^{n+1} \rightarrow \mathbb{R}^{n}$ be defined by $p(\mathbf{x})=\left(x_{1}, \ldots, x_{n}\right)$, i.e., $p$ ignores the last coordinate.

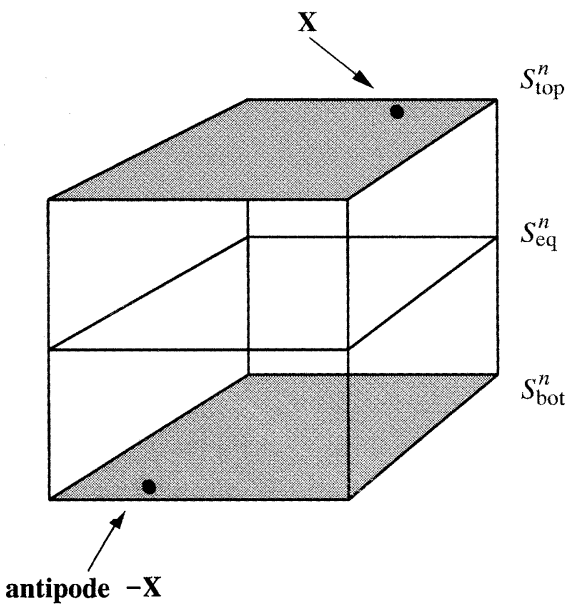

Figure 1. Top, bottom, and equator of the cubical $n$-sphere (here $n=2$ ). An example of antipodal points is indicated.

For all $\mathbf{x}$ in $S_{\text {top }}^{n}$, define $g(\mathbf{x})=p(\mathbf{x})-f(p(\mathbf{x}))$. For all $\mathbf{x}$ in $S_{\text {bot }}^{n}$, define $g(\mathbf{x})=$ $p(\mathbf{x})+f(-p(\mathbf{x}))$.

Since $p(-\mathbf{x})=-p(\mathbf{x})$, one may check that $g(-\mathbf{x})=-g(\mathbf{x})$. Thus $g$ is, so far, antipode-preserving. It is continuous, since $f$ and $p$ are. If $g(\mathbf{x})=\mathbf{0}$ then $p(\mathbf{x})$ is a fixed point for $f$.

Now we want to define $g$ on the "side" faces of the cubical $n$-sphere so that it matches up continuously with $g$ on $S_{\text {top }}^{n}$ and $S_{\text {bot }}^{n}$ and is still antipode-preserving, but is never zero on the sides. The latter is the tricky part.

One might try to extend the values of $g$ linearly from top to bottom, but this does not guarantee that $g \neq \mathbf{0}$ on the sides. However, the following lemmas show that if we define $g$ suitably on the equator, we can linearly extend the values from the equator to top and bottom without creating a new zero for $g$.

Lemma 1. Let $F$ be a "side" face of the cubical $S^{n}$. That is, there exists some $k$, $1 \leq k \leq n$, such that for all $\mathbf{x}$ in $F, x_{k}$ is constant and equal to +1 or -1 . Then for 
all $\mathbf{x}$ in $F \cap\left(S_{\text {bot }}^{n} \cup S_{\text {top }}^{n}\right)$, the coordinate function $g_{k}(\mathbf{x})$ is either 0 or has the same sign as $x_{k}$.

Proof: By the definition of $g$, on $F \cap S_{\text {top }}^{n}, g_{k}(\mathbf{x})=x_{k}-f_{k}(p(\mathbf{x}))$ and on $F \cap S_{\text {bot }}^{n}$, $g_{k}(\mathbf{x})=x_{k}+f_{k}(-p(\mathbf{x}))$. Since $f$ is a map to an $n$-cube, $\left|f_{k}\right| \leq 1$. Hence, if $x_{k}=1$, then $g_{k}(\mathbf{x}) \geq 0$ for all $\mathbf{x} \in F \cap\left(S_{\text {bot }}^{n} \cup S_{\text {top }}^{n}\right)$. If $x_{k}=-1$, then $g_{k}(\mathbf{x}) \leq 0$ for all $\mathbf{x} \in F \cap\left(S_{\text {bot }}^{n} \cup S_{\text {top }}^{n}\right)$.

Now let $S_{e q}^{n}$ denote the "equator" of $S^{n}$, i.e., $\left\{\mathbf{x} \in S^{n} \mid \mathbf{x}=\left(x_{1}, \ldots, x_{n}, 0\right)\right\}$. For all $\mathbf{x} \in S_{e q}^{n}$, define $g$ on $S_{e q}^{n}$ by

$$
g(\mathbf{x})=p(\mathbf{x})+\frac{g\left(x_{1}, \ldots, x_{n},-1\right)+g\left(x_{1}, \ldots, x_{n}, 1\right)}{2},
$$

and observe that $g$ is now antipode-preserving on the equator.

Lemma 2. For all $\mathbf{x} \in S_{e q}^{n}$, if $\left|x_{k}\right|=1$, then the coordinate function $g_{k}(\mathbf{x})$ is not 0 and has the same sign as $x_{k}$.

Proof: Lemma 1 shows that if $\left|x_{k}\right|=1, g_{k}$ has the same sign as $x_{k}$ on the top and bottom faces if it is not zero. Therefore, using (1) and $p_{k}(\mathbf{x})=x_{k}= \pm 1$, we see that $g_{k}(\mathbf{x})$ is non-zero on the equator and has the same sign as $x_{k}$.

To define $g$ on the equator we have "averaged" the values of the corresponding points on $S_{\text {top }}^{n}$ and $S_{\text {bot }}^{n}$ and then "lifted" that average by $p(\mathbf{x})$ (which equals $x_{k}$ in
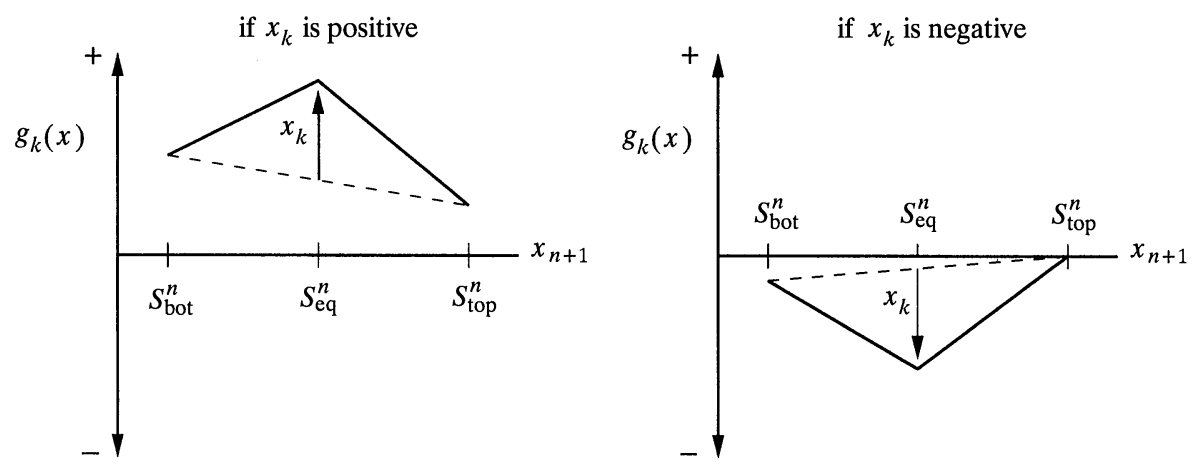

Figure 2. Extending the values of $g_{k}$ linearly from the equator to top and bottom. Graphs show a cross-section of $g_{k}$ values along on "longitude" of the cubical $n$-sphere on the faces determined by $x_{k}=+1,-1$.

the $k$-th coordinate) to pull it away from possibly being zero. See Figure 2.

We now define $g$ continuously on the rest of $S^{n}$ by extending it linearly from the equator to the values on $S_{\text {bot }}^{n}$ and $S_{\text {top }}^{n}$. That is, for $0 \leq x_{n+1} \leq 1$, let

$$
g(\mathbf{x})=x_{n+1} g\left(x_{1}, \ldots, x_{n}, 1\right)+\left(1-x_{n+1}\right) g\left(x_{1}, \ldots, x_{n}, 0\right) .
$$

For $-1 \leq x_{n+1} \leq 0$, let

$$
g(\mathbf{x})=-x_{n+1} g\left(x_{1}, \ldots, x_{n},-1\right)+\left(1+x_{n+1}\right) g\left(x_{1}, \ldots, x_{n}, 0\right) .
$$

Refer to Figure 2 again. Note that $g$ is continuous and antipode-preserving. 
Furthermore, it can achieve $\mathbf{0}$ only on $S_{\text {top }}^{n}$ or $S_{\text {bot }}^{n}$, because of

Lemma 3. If $\left|x_{n+1}\right|<1$, then $g(\mathbf{x}) \neq \mathbf{0}$.

Proof: Since $\left|x_{n+1}\right|<1$, we are on a side face and therefore there exists some $k$, $1 \leq k \leq n$, for which $x_{k}= \pm 1$. We shall show that $g(\mathbf{x})$ cannot be zero by showing that the coordinate function $g_{k}(\mathbf{x})$ is non-zero.

Consider (2) and (3). By Lemmas 1 and 2, $g_{k}\left(x_{1}, x_{2}, \ldots, x_{n}, \pm 1\right)$ and $g_{k}\left(x_{1}, x_{2}, \ldots, x_{n}, 0\right)$ have the same sign as $x_{k}$, and the latter is non-zero. Moreover, $\left(1-x_{n+1}\right)$ and $\left(1+x_{n+1}\right)$ are strictly positive because $\left|x_{n+1}\right|<1$. Equations (2) and (3) now imply that $g_{k}(\mathbf{x})$ is non-zero and, in fact, has the same sign as $x_{k}$.

Now that $g$ is defined everywhere on $S^{n}$, the Borsuk-Ulam Theorem implies that there exists a pair $\{\mathbf{x},-\mathbf{x}\}$ such that $g(\mathbf{x})=g(-\mathbf{x})$. But $g(\mathbf{x})=-g(-\mathbf{x})$, since $g$ is antipode-preserving. Therefore $g(\mathbf{x})=g(-\mathbf{x})=\mathbf{0}$ which, by Lemma 3, implies that one of the pair $\{\mathbf{x},-\mathbf{x}\}$ is in $S_{\text {top }}^{n}$. Without loss of generality, suppose it is $\mathbf{x}$. Then $g(\mathbf{x})=p(\mathbf{x})-f(p(\mathbf{x}))=\mathbf{0}$ on $S_{\text {top }}^{n}$ implies that for $y=p(\mathbf{x}) \in B^{n}$, we have $f(\mathbf{y})=\mathbf{y}$, which proves the Brouwer Fixed Point Theorem.

ACKNOWLEDGMENTS. I thank Michael Starbird for many lively discussions and Courtney Coleman for helpful stylistic suggestions.

\section{REFERENCES}

1. K. Borsuk, Drei Sätze über die $n$-dimensionale euklidische Sphäre, Fund. Math., 20 (1933), 177-190.

2. L.E.J. Brouwer, Über Abbildung von Mannigfaltigkeiten, Math. Ann., 71 (1912), 97-115.

3. J. Dugundji and A. Granas, Fixed Point Theory, Polish Scientific Publishers, Warsaw, 1982.

4. W.S. Massey, A Basic Course in Algebraic Topology, Springer-Verlag, New York, 1991.

5. J. Rotman, An Introduction to Algebraic Topology, Springer-Verlag, New York, 1984.

6. E.H. Spanier, Algebraic Topology, Springer-Verlag, New York, 1966.

\section{Department of Mathematics}

Harvey Mudd College

Claremont, $C A 91711$

su@math.hmc.edu 